\title{
Brief Report \\ Correction of an Orthogonal Procrustes Rotation Procedure Described by Guilford and Hoepfner
}

\author{
Jos M. F. ten Berge \\ University of Groningen
}

\begin{abstract}
Index terms: factor matching, least-squares rotation, target rotation.
\end{abstract}

In their book The Analysis of Intelligence, Guilford and Hoepfner (1971, pp. 52-53) described Cliff's method of rotating a principal-factor matrix to a best least-squares fit with a hypothesized target factor matrix. This note shows that Guilford and Hoepfner's description of Cliff's method is incorrect.

The rotation method related by Guilford and Hoepfner is the following: Let

$\mathbf{F}=$ the empirical factor matrix (of principal factors)

$\mathbf{A}=$ the target matrix

$\hat{\mathbf{A}}=$ the least-squares estimate of the target matrix

$\mathbf{T}=$ unknown transformation of $\mathbf{F}$ to $\hat{\mathbf{A}}$

$\mathbf{B}=\mathbf{A}^{\prime} \mathbf{F}$

$\mathbf{B B}^{\prime}=\mathbf{C}$

$\mathbf{D}=r \times r$ matrix whose columns are eigenvectors of $\mathbf{C}$

$\mathbf{B}^{\prime} \mathbf{D}=\mathbf{E}$

$\mathbf{T}=\mathbf{E}\left[\operatorname{Diag}\left(\mathbf{E}^{\prime} \mathbf{E}\right)\right]^{-1 / 2}$ $\mathbf{F T}=\hat{\mathbf{A}}$

To verify that the resulting $\mathbf{T}$ is not the optimal $\mathrm{T}^{*}$ that results from using Cliff's method, an outline is presented of how $\mathbf{T}^{*}$ must be computed.

Let $\mathbf{A}^{\prime} \mathbf{F}=\mathbf{B}$ have the singular value or EckartYoung decomposition

$\mathbf{A}^{\prime} \mathbf{F}=\mathbf{U \delta V}$.

APPLIED PSYCHOLOGICAL MEASUREMENT

Vol. 13, No. 1, March 1989, pp. 105-106

(C) Copyright 1989 Applied Psychological Measurement Inc. 0146-6216/89/010105-02\$1.35
Then $\mathbf{T}^{*}$ must be computed as

$\mathbf{T}^{*}=\mathbf{V}^{\prime} \mathbf{U}^{\prime}$

(Cliff, 1966, pp. 35-36). Guilford and Hoepfner (1971) defined $\mathbf{D}$ as an eigenvector matrix of

$\mathbf{C}=\mathbf{B B}^{\prime}=\mathbf{A}^{\prime} \mathbf{F F}^{\prime} \mathbf{A}=\mathbf{U}^{2} \mathbf{U}^{\prime}$,

which implies $\mathbf{D}=\mathbf{U}$. Next, Guilford and Hoepfner computed

$\mathbf{E}=\mathbf{B}^{\prime} \mathbf{D}=\mathbf{F}^{\prime} \mathbf{A D}=\mathbf{V}^{\prime} \boldsymbol{\delta} \mathbf{U}^{\prime} \mathbf{U}=\mathbf{V}^{\prime} \boldsymbol{\delta}$

to arrive at

$$
\begin{aligned}
\mathbf{T} & =\mathbf{E}\left[\operatorname{Diag}\left(\mathbf{E}^{\prime} \mathbf{E}\right)\right]^{-1 / 2} \\
& =\mathbf{V}^{\prime} \boldsymbol{\delta}\left[\operatorname{Diag}\left(\boldsymbol{\delta} \mathbf{V}^{\prime} \boldsymbol{\delta}\right)\right]^{-1 / 2} \\
& =\mathbf{V}^{\prime} \boldsymbol{\delta}\left(\boldsymbol{\delta}^{2}\right)^{-1 / 2} \\
& =\mathbf{V}^{\prime},
\end{aligned}
$$

which shows that $\mathbf{T}$ differs from $\mathbf{T}^{*}$ in that

$\mathbf{T}^{*}=\mathbf{T U}^{\prime}=\mathbf{T D}^{\prime}$.

Thus, Guilford and Hoepfner have presented an erroneous description of Cliff's method. Specifically, the matrix $\mathbf{T}$ computed according to their description of Cliff's method still must be postmultiplied by $\mathbf{D}^{\prime}$ to arrive at the correct solution.

\section{Computational Example}

Let a $4 \times 2$ principal-factor matrix $\mathbf{F}$ and the hypothesized target $\mathbf{A}$, constructed from $\mathbf{F}$, be as follows:

$\mathbf{F}=\left(\begin{array}{rr}.8 & .4 \\ .8 & .4 \\ .8 & -.2 \\ .8 & -.6\end{array}\right)$ 
and

$\mathrm{A}=\left(\begin{array}{cc}.8 & 0 \\ .8 & 0 \\ 0 & .68 \\ 0 & 1.00\end{array}\right)$

thus giving

$\mathbf{B}=\mathbf{A}^{\prime} \mathbf{F}=\left(\begin{array}{rr}1.280 & .640 \\ 1.344 & -.736\end{array}\right)$

and

$\mathbf{B B}^{\prime}=\mathbf{C}=\left(\begin{array}{ll}2.0480 & 1.2493 \\ 1.2493 & 2.3480\end{array}\right)$.

The eigenvectors of $\mathbf{C}$ are in the matrix

$\mathbf{D}=\left(\begin{array}{rr}.6636 & .7481 \\ .7481 & -.6636\end{array}\right)$,

which implies that

$\mathbf{F}^{\prime} \mathbf{A D}=\mathbf{E}=\left(\begin{array}{rr}1.85485 & .06569 \\ -.12590 & .96719\end{array}\right)$.

Normalizing $\mathbf{E}$ yields

$$
\begin{aligned}
\mathbf{T} & =\mathbf{E}\left[\operatorname{Diag}\left(\mathbf{E}^{\prime} \mathbf{E}\right)\right]^{-1 / 2} \\
& =\left(\begin{array}{rr}
.9977 & .0678 \\
-.0678 & .9977
\end{array}\right),
\end{aligned}
$$

and hence

$\hat{\mathbf{A}}=\mathbf{F T}=\left(\begin{array}{lc}.771 & .453 \\ .771 & .453 \\ .812 & -.145 \\ .8388 & -.5444\end{array}\right)$

It can be seen that $\hat{\mathbf{A}}$ does not approximate the target very well. To obtain the best least-squares fit, $\hat{\mathbf{A}}$ must be postmultiplied by $\mathbf{U}^{\prime}=\mathbf{D}^{\prime}$. This yields Cliff's solution

$\hat{\mathbf{A}} \mathbf{D}^{\prime}=\left(\begin{array}{ll}.8505 & .276 \\ .8505 & .276 \\ .4304 & .7037 \\ .1494 & .9888\end{array}\right)$

which does approximate $\mathbf{A}$. To check the optimality of $\hat{\mathbf{A}} \mathbf{D}^{\prime}$, compute
$\mathbf{A}^{\prime} \hat{\mathbf{A}} \mathbf{D}^{\prime}=\left(\begin{array}{rr}1.3608 & .4416 \\ .4421 & 1.4673\end{array}\right)$,

which is, within limits of computational accuracy, symmetric and positive semi-definite; hence the best least-squares fit has been obtained with $\hat{\mathbf{A}} \mathbf{D}^{\prime}$ (ten Berge, 1977, Theorem 2).

A reflection of the sign of the second column of $\hat{\mathbf{A}}$ would improve the fit of $\hat{\mathbf{A}}$. Such a reflection is permitted because the eigenvectors of $\mathbf{C}$ are only determined up to a reflection. However, this still leaves the Guilford-Hoepfner solution without leastsquares optimality.

\section{Conclusions}

Clearly, the Guilford-Hoepfner description of Cliff's method is incorrect. Although this might imply that all target rotations done by Guilford and Hoepfner (1971) are incorrect, a reanalysis of several of their matrices yielded results that agreed with Cliff's method. These results, therefore, indicate that they actually used Cliff's method for the analysis of their intelligence studies, although they erroneously described the method in their book.

\section{References}

Cliff, N. (1966). Orthogonal rotation to congruence. Psychometrika, 31, 33-42.

Guilford, J. P., \& Hoepfner, R. (1971). The analysis of intelligence. New York: McGraw-Hill.

ten Berge, J. M. F. (1977). Orthogonal Procrustes rotation for two or more matrices. Psychometrika, 42, 267-276.

\section{Author's Address}

Send requests for reprints or further information to $\mathrm{J}$. M. F. ten Berge, Department of Psychology, University of Groningen, Grote Markt 32, 9712 HV Groningen, The Netherlands. 